\title{
Epistemic logic without closure
}

\author{
Stephan Leuenberger ${ }^{1}\left(\mathbb{D} \cdot\right.$ Martin Smith $^{2}$
}

Received: 25 October 2018 / Accepted: 14 August 2019 / Published online: 27 August 2019 (c) The Author(s) 2019

\begin{abstract}
All standard epistemic logics legitimate something akin to the principle of closure, according to which knowledge is closed under competent deductive inference. And yet the principle of closure, particularly in its multiple premise guise, has a somewhat ambivalent status within epistemology. One might think that serious concerns about closure point us away from epistemic logic altogether-away from the very idea that the knowledge relation could be fruitfully treated as a kind of modal operator. This, however, need not be so. The abandonment of closure may yet leave in place plenty of formal structure amenable to systematic logical treatment. In this paper we describe a family of weak epistemic logics in which closure fails, and describe two alternative semantic frameworks in which these logics can be modelled. One of these-which we term plurality semantics - is relatively unfamiliar. We explore under what conditions plurality frames validate certain much-discussed principles of epistemic logic. It turns out that plurality frames can be interpreted in a very natural way in light of one motivation for rejecting closure, adding to the significance of our technical work. The second framework that we employ—neighbourhood semantics - is much better known. But we show that it too can be interpreted in a way that comports with a certain motivation for rejecting closure.
\end{abstract}

Keywords Epistemic logic $\cdot$ Closure $\cdot$ Compartmentalisation · Fragmentation · Risk minimisation $\cdot$ Neighbourhood semantics $\cdot$ Plurality semantics

\footnotetext{
Many thanks to audiences at the Glasgow-Melbourne Formal Philosophy Workshop, and the University of Oslo's workshop on Philosophical Applications of Modal Logic, in particular Peter Fritz, Wesley Holliday, Shawn Standefer, and Alan Weir. For comments on earlier drafts, we are grateful to Bruno Whittle and to our anonymous referees. This work was supported by the Arts and Humanities Research Council [Grant Nos. AH/M009610/1, AH/J004189/1].
}

Stephan Leuenberger

stephan.leuenberger@glasgow.ac.uk

Martin Smith

martin.smith@ed.ac.uk

1 School of Humanities, University of Glasgow, Glasgow G12 8QQ, UK

2 School of Philosophy, Psychology and Language Sciences, University of Edinburgh, Edinburgh EH8 9AD, UK 


\section{Closure in epistemic logic}

The language of epistemic logic is an extension of the language of ordinary propositional logic with an added operator $K$, usually read ' $S$ knows that ...', ' $S$ is in a position to know that ...', ' $S$ implicitly knows that ...', '... follows from what $S$ knows' [see Fagin et al. (1995), Williamson (2000, Sect. 10.4)]. The behaviour of this operator is generally taken to be governed by one of the normal modal logics and modelled, using the Kripke semantic framework, as a kind of universal quantifier over possible worlds. Proposed logics have included KT (Williamson 2000, Sect. 10.4), S4 (Hintikka 1962), S4.2 (Lenzen 1978; Stalnaker 2006), S4.3 (van der Hoek 1996), S4.4 (von Kutschera 1976) and S5 (Fagin et al. 1995). Sometimes additional operators are added in order to represent the knowledge of further agents. We will only consider single agent systems here, though the logics we describe could certainly be extended to multiple agent systems. If we interpret $K$ to mean ' $S$ has justification for believing that ...' or ' $S$ is in a position to justifiably believe that . .', we arrive at a different kind of epistemic logic — a logic of justification (Rosenkranz 2018; Smith 2018). Though our primary focus here will be on the logic of knowledge, we will return to the logic of justification from time to time.

Epistemic closure principles are designed to capture the idea that deduction is a sure-fire way of extending one's corpus of knowledge - that drawing deductively valid inferences can never take us from the realm of knowledge into the realm of pure guesswork. While this basic idea is undoubtedly attractive, it has proved somewhat resistant to precise formulation. There are, indeed, a number of subtly different versions of the closure principle on the market [see, for instance, Kvanvig (2006)]. For the most part, the issue of selecting the most plausible or defensible version need not concern us here. The kind of closure principles that are part of epistemic logic are closest to the formulation used by Cohen (2002, p. 312) ("If S knows P and S knows P entails $\mathrm{Q}$, then $\mathrm{S}$ knows (or at least is in a position to know) Q") and to the closure principle (4) in Kvanvig (2006) ("If you know P, and know that P entails Q, then you are in a position to know Q").

The first thing to observe when considering the status of closure within epistemic logic is that the following inference pattern will be admissible in all of the epistemic logics listed above and, indeed, admissible in any normal epistemic logic.

[MPC] For any positive integer $n$,

$\vdash\left(\phi_{1} \wedge \cdots \wedge \phi_{n}\right) \supset \psi$

$\vdash\left(K \phi_{1} \wedge \cdots K \phi_{n}\right) \supset K \psi$

MPC (for multiple premise closure) states that if $\left(\phi_{1} \wedge \cdots \wedge \phi_{n}\right) \supset \psi$ is a logical theorem (if $\phi_{1} \wedge \cdots \wedge \phi_{n}$ deductively entails $\left.\psi\right)$ then $\left(K \phi_{1} \wedge \cdots \wedge K \phi_{n}\right) \supset K \psi$ is a logical theorem $\left(K \phi_{1} \wedge \cdots \wedge K \phi_{n}\right.$ deductively entails $\left.K \psi\right)$.

We can also set out a weaker single premise version of the principle: ${ }^{1}$

[SPC]

$$
\frac{\vdash \phi \supset \psi}{\vdash K \phi \supset K \psi}
$$

\footnotetext{
${ }^{1}$ On the face of it, the informal characterisations of closure we quoted only suggest this weaker principle.
} 
SPC states that if $\phi \supset \psi$ is a logical theorem (if $\phi$ deductively entails $\psi$ ) then $K \phi \supset K \psi$ is a logical theorem ( $K \phi$ deductively entails $K \psi)$.

If $K$ is to be read ' $S$ knows that ...', in line with the first suggestion above, then these patterns would appear to enshrine closure principles so strong that almost no-one would be inclined to accept them in full generality. The idea that one automatically knows the deductive consequences of one's knowledge is surely unrealistic for all but the most idealised of agents. Knowledge is generally thought to require belief but, quite clearly, an ordinary epistemic agent could fail to believe the deductive consequences of things that she knows - she could simply fail to perform or even consider the relevant deductions.

If $K$ is given any of the alternative readings noted above (each of which are similar in spirit) then the principles enshrined by SPC and MPC become altogether more plausible. If $K$ is read ' $\mathrm{S}$ is in a position to know that ...' then what these patterns require is that one be in a position to know the deductive consequences of things that one is in a position to know. In other words, the relation of being in a position to know is closed under the multi-premise/single-premise deductive consequence relation. We shall adopt this as our official reading of the $K$ operator. To be in a position to know something, in the relevant sense, it is not enough that one be psychologically capable of knowing it. Neither is it enough that one could feasibly acquire knowledge by conducting some investigation or inquiry into the matter. Rather, to be in a position to know something, the fact must lie open and accessible to one's mind, such that one could know it without needing to gather any further evidence [see Williamson (2000, Sect. 4.2)].

For a time controversy over closure principles focused upon the role that such principles play in certain well known sceptical arguments [see, for instance, Dretske (1970), Dretske (2005), Nozick (1981)]. Many ordinary, mundane premises deductively entail the negations of sceptical hypotheses. But it's intuitive to think that we cannot know the negations of sceptical hypotheses in which case, if closure holds, we cannot know the ordinary mundane premises either. The discussion of these sceptical arguments has, for the most part, run its course and the prevailing view, we think, is that resisting scepticism need not ultimately require the abandonment of closure. In any case, we will focus here upon two rather different concerns about closure, both of which have been the focus of more recent attention.

The first of these concerns arises against the background of a view on which knowledge requires the minimisation, but not the complete elimination, of falsity risk. It is often observed that deductive inferences that involve multiple premises can serve to aggregate the risk of falsity - the conclusion may run a higher falsity risk than any of the premises taken individually. In particular, the conclusion may run such a high falsity risk as to preclude one from being in a position to know it, even if none of the premises do. In Knowledge and Lotteries, John Hawthorne puts the point in this way:

Deductive inference from multiple premises aggregates risks. The risk accruing to one's belief in each premise may be small enough to be consistent with the belief having the status of knowledge. But the risks may add up, so that the deduced belief may be in too great a danger of being false to count as knowledge. [Hawthorne (2004, p. 47)] 
It's important to note that the risk aggregation motivation for rejecting closure, unlike the anti-sceptical motivation, appears to leave the single premise version of the principle intact. Single premise deductive inferences cannot, after all, aggregate risk in the same way as multiple premise deductive inferences. In a single premise deductive inference, the falsity of the conclusion guarantees the falsity of the premise, in which case the risk that the conclusion is false cannot possibly exceed the risk that the premise is false. As Hawthorne puts it ' . . deductive inference from a single premise does not seem like a candidate for risky inference. If $p$ entails $q$ then $q$ must be logically weaker or equivalent to $p$ ' [Hawthorne (2004, p. 47)]. ${ }^{2}$ The risk minimisation theory provides reason to reject MPC but no reason to reject SPC. If we take risk minimisation to be necessary for justification then we also have reason to reject MPC on the justification reading of the $K$ operator.

The second concern about multiple premise closure arises against the background of a view on which an agent's body of knowledge is compartmentalised-divided into autonomous compartments that may not be fully integrated with one another. In 'Elusive Knowledge' David Lewis mentions the anti-sceptical motivation for rejecting closure, which he associates with Dretske, and then continues:

There is another reason, different from Dretske's, why we might doubt closure. Suppose two or more premises jointly imply a conclusion. Might not someone who is compartmentalized in his thinking - as we all are-know each of the premises but fail to bring them together in a single compartment? Then might he not fail to know the conclusion? Yes; and I would not like to plea idealizationof-rationality as an excuse for ignoring such cases. [Lewis (1996, p. 565)]

On this view, it is compartments, rather than agents, that serve as the primary subjects of potential knowledge. An agent might be said to be in a position to know something only in a derivative sense-iff one of his or her underlying compartments is [Lewis (1996, p. 443)].

Closure will fail, on this sort of picture, in so far as compartments can fail to share their potential knowledge with one another. Just because one compartment is in a position to know one premise of a deductive inference and another compartment is in a position to know another, there need not be any compartment that is in a position to know the conclusion. Like the risk minimisation motivation for rejecting closure, the compartmentalisation motivation poses no obvious threat to single premise closure. After all, single premise deductive inferences do not require the integration of potential knowledge from different compartments. The compartmentalisation theory provides reason to reject MPC, but no reason to reject SPC. If compartments can fail to share their potential justification with one another we also have reason to reject MPC on the justification reading of the $K$ operator. ${ }^{3}$ Something close to the compartmentalisation

\footnotetext{
2 For a dissenting view, on which risk aggregation considerations can pose a threat even to single premise closure, see Lasonen-Aarnio (2008). For discussion of this view, see Smith (2013), Tang (2018).

3 This is certainly the case on the ' $\mathrm{S}$ is in a position to justifiably believe ...' reading of the operator. Just because one compartment is in a position to justifiably believe one premise of a deductive inference and another compartment is in a position to justifiably believe another, there need not be any compartment that is in a position to justifiably believe the conclusion. On some views, however, having justification for believing a proposition does not entail that one is in a position to justifiably believe it-having justification
} 
motivation for rejecting MPC is noted by Fagin and Halpern (1987), who suggest that what subjects believe or know is relative to different 'frames of mind' that one cannot occupy simultaneously. ${ }^{4}$

Lewis says little about what compartments actually are and why we should accept their existence, and we won't attempt to elaborate here [for some related discussion see Lewis (1982), Stalnaker (1984, Chap. 5) Spectre (2018)]. For present purposes, it is enough that compartments be thought of as partially disjoint bodies of potential knowledge. Even if one is inclined to doubt that individuals' minds are divided into compartments in this way, the foregoing reasoning may still pose a threat to MPC taken in full generality. As well as attributing knowledge to individuals, we sometimes describe communities, corporations, nations etc. as 'knowing' things. ${ }^{5}$ Such talk may be metaphorical—but if we take it literally then there will be subjects of knowledge that are transparently compartmentalised in just the sense at play here. If a group is in a position to know something whenever some of its members are, then the potential knowledge of that group need not be closed under multiple premise deductive consequence. We won't pursue this further here-though we will have a little more to say about group knowledge along the way. It is not our aim, in any case, to defend the rejection of multiple premise closure-our aim is to develop epistemic logics that do justice to this rejection and to the kinds of perspectives (right or wrong) that might motivate it.

The MPC inference pattern can, we might observe, be decomposed into the weaker SPC pattern and the following theorem schema:

\section{(A) $(K \phi \wedge K \psi) \supset K(\phi \wedge \psi)$}

(A) can be thought of as an agglomeration principle stating that, if one is in a position to know each conjunct of a conjunction then one is in a position to know the conjunction. SPC is, evidently, a limiting case of MPC (for which $n=1$ ). Proving (A) using MPC is very simple: as a tautology, $(\phi \wedge \psi) \supset(\phi \wedge \psi)$ is a theorem, and by MPC, so is (A). MPC can, in turn, be derived using (A) and SPC. ${ }^{6}$ (A), in effect, allows us to collapse multiple premise deductive entailments with known premises, into single premise deductive entailments with a known premise.

Both the risk minimisation and compartmentalisation theories would appear to pinpoint (A) as the source of trouble in MPC. It is the conjoining of individually known premises that can allow falsity risk to aggregate and can require the integration of potential knowledge from separate compartments. Once all of the premises are conjoined, no further risk is introduced and no further integration is required by the deductive transition to a conclusion. Both the risk minimisation and compartmentalisation theories motivate the rejection of MPC, via the rejection of (A), and the

\section{Footnote 3 continued}

for believing a proposition is compatible with one being blocked, for one reason or another, from exploiting that justification in forming a justified belief [Smithies (2012, Sect. 4)]. As such, the compartmentalisation theory may leave some scope for preserving MPC for the 'S has justification for believing ...' reading of the operator. We won't pursue this further here.

4 Compartmentalisation may be a feature of theories as well as thinkers; see Brown and Priest (2004), who allow for a limited aggregation across compartments.

5 See Bird (2014) and Tuomela (2004), for example.

6 See the proof of Theorem 4.3(3) in Chellas (1980, p. 115) (with 'C' for our '(A)' and 'RM' for our 'SPC'). 
retention of SPC. Though we will briefly consider some epistemic logics in which even SPC fails, we are primarily interested in logics that complement this particular combination of views - in logics that are located "in between" SPC and MPC.

\section{Kripke semantics}

In developing these logics, we begin by setting things up in a more formal fashion. Let the language $\mathcal{L}$ include, in its vocabulary, countably many sentential constants $\left(P_{0}, P_{1}, P_{2}, P_{3}, \ldots\right)$, the verum and falsum constants ( $T$ and $\perp$ ), the extensional operators $(\wedge, \vee, \sim$ and $\supset)$, the operator $K$ and punctuation. The sentences of $\mathcal{L}$ can be built using the standard recursive clauses for operators and punctuation. We will continue to use lower case Greek letters as metalinguistic sentence variables and use \& , ᄀ and $\rightarrow$ as the extensional operators of the metalanguage.

The following nine principles serve as the building blocks of the most familiar modal logics:

$$
\begin{aligned}
& K(\phi \supset \psi) \supset(K \phi \supset K \psi) \\
& K \phi \supset \sim K \sim \phi \\
& K \phi \supset \phi \\
& \sim K \sim K \phi \supset \phi \\
& K \phi \supset K K \phi \\
& \sim K \sim K \phi \supset K \sim K \sim \phi \\
& K(K \phi \supset K \psi) \vee K(K \psi \supset K \phi) \\
& \phi \supset(\sim K \sim K \phi \supset K \phi) \\
& \sim K \phi \supset K \sim K \phi
\end{aligned}
$$

A K-logic includes all truth functional tautologies, all instances of (K), and is closed under two basic inference rules-modus ponens and what is sometimes called 'knowledge generalisation':

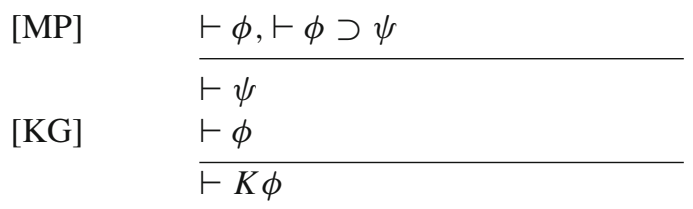

The minimal K-logic is simply called $\mathbf{K}$. Further $\mathbf{K}$-logics that have been considered result from adding all instances of some selection (zero or more) of the remaining principles. The weakest $\mathbf{K}$-logic that might be taken seriously as a potential logic of knowledge is the logic KT. (T), of course, captures the idea that knowledge is factive and, as such, one can only be in a position to know truths. If the $K$ operator is given a justification reading then $(\mathrm{T})$ is no longer appropriate and should, perhaps, be replaced by (D). This is one significant difference between the logic of knowledge and the logic of justification. Much of the philosophical literature on epistemic logic has focussed upon the viability of the remaining principles_-particularly the 'positive introspection' or 'KK' principle (4) and the 'negative introspection' principle (5). If, of course, concerns about closure are well founded then no $\mathbf{K}$-logic is cut out to serve as an adequate epistemic logic. With the two inference rules in place, $(\mathrm{K})$ already 
provides everything that we need in order to derive full MPC. Since any instance of (K) can also be derived using MPC and the other inference rules we could simply substitute the admissibility of MPC for the presence of the (K) axiom schema. ${ }^{7}$ That is, a K-logic could simply be defined as a logic that includes all truth functional tautologies and admits the rules MP, KG and MPC.

A Kripke frame $\mathcal{F}$ is a pair $\langle W, R\rangle$ where $W$ is a nonempty set of possible worlds, and $R$ is a binary relation upon $W$. A Kripke model $\mathcal{M}$ based upon the frame $\langle W, R\rangle$ is a triple $\langle W, R, I\rangle$ where $I$ is a valuation, mapping the non-negative integers to subsets of $W$ (i.e. mapping $i$ to the set of worlds where the atomic sentence $P_{i}$ is true in the model). We define recursively what it is for a sentence $\phi$ to be true at a world $w$ in a Kripke model $\mathcal{M}-\models_{w}^{\mathcal{M}} \phi$ :

$$
\begin{aligned}
& \vDash{ }_{w}^{\mathcal{M}} \top \\
& \not \forall_{w}^{\mathcal{M}} \perp \\
& \vDash{ }_{w}^{\mathcal{M}} P_{n} \text { iff } w \in I(n) \\
& \vDash_{w}^{\mathcal{M}} \phi \wedge \psi \text { iff } \models_{w}^{\mathcal{M}} \phi \& \vDash_{w}^{\mathcal{M}} \psi \\
& \vDash_{w}^{\mathcal{M}} \sim \phi \text { iff } \not \nvdash_{w}^{\mathcal{M}} \phi \\
& \models_{w}^{\mathcal{M}} K \phi \text { iff } \forall x \in W, \quad w R x \rightarrow \vDash_{x}^{\mathcal{M}} \phi
\end{aligned}
$$

According to the Kripke-semantic clause for $K, K \phi$ is true at a world $w$ in a Kripke model iff $R$ connects $w$ only to worlds at which $\phi$ is true.

A sentence $\phi$ might be described as valid relative to a Kripke model $\mathcal{M}=\langle W, R, I\rangle$ just in case for every $w \in W \models_{w}^{\mathcal{M}} \phi$. In this case we write $\models^{\mathcal{M}} \phi$. A sentence might be described as valid relative to a Kripke frame $\mathcal{F}=\langle W, R\rangle$ just in case it is valid relative to any model based upon that frame. In this case we write $\models^{\mathcal{F}} \phi$. Finally, a sentence might be described as valid relative to a class of Kripke frames just in case it is valid with respect to every frame in the class.

The relation $R$ that features in the clause for $K$ can be interpreted in a range of different ways. At a minimum, what it means for world $w_{1}$ to stand in relation $R$ to world $w_{2}\left(w_{1} R w_{2}\right)$ is for $w_{2}$ to be consistent with everything that $S$ is in a position to know at $w_{1}$ - just in case, for all that $S$ is in a position to know at $w_{1}$, she may be at $w_{2}$. Understanding $R$ in this way will, of course, render the clause for $K$ viciously circular, if considered as a putative analysis of knowledge-on this interpretation, what the clause says is that $S$ is in a position to know a sentence $\phi$ at a world $w$ iff $\phi$ is true at all worlds that are consistent with what $S$ is in a position to know at $w$. But the clause need not be intended as part of a philosophical analysis. Rather, it provides us with a new way of representing information about what $S$ is in a position to know-representing it in terms of a binary relation on worlds.

As is well known, the logic $\mathbf{K}$ is determined by the class of Kripke frames. That is, a sentence is a theorem of $\mathbf{K}$ iff it is valid in all Kripke frames. By placing constraints upon the relation $R$, we can limit the class of permissible frames and determine various stronger logics. Each of the optional principles listed above corresponds to a distinctive constraint upon $R$ :

\footnotetext{
${ }_{7}$ For details, see the proof of Theorem 4.3(1) in Chellas (1980, p. 115) ('KG' corresponds to 'RN', and 'MPC' to 'RK').
} 


\section{Principle Constraint}

(D)
$\langle\boldsymbol{W}, \boldsymbol{R}\rangle$ is ...
serial
reflexive
symmetric
transitive
convergent
connected
triangular
Euclidean

The logics that result from combining any of these optional principles will be determined by the class of Kripke frames meeting the corresponding constraints.

Given the understanding of $R$ set out above, the content of at least some of these constraints can be made apparent. Reflexivity, for instance, enforces the idea that a world $w$ is always consistent with what $S$ is in a position to know at $w$. Symmetry requires that if $w_{2}$ is consistent with everything that $S$ is in a position to know at $w_{1}$ then $w_{1}$ must be consistent with everything that $S$ is in a position to know at $w_{2}$. Transitivity requires that, if $w_{3}$ is consistent with everything that $S$ is in a position to know at $w_{2}$ but not consistent with everything that $S$ is in a position to know at $w_{1}$ then $w_{2}$ is not consistent with everything that $S$ is in a position to know at $w_{1}$. In effect, if the potential knowledge that $S$ possesses at $w_{2}$ is, in some respect, weaker than the potential knowledge that $S$ possesses at $w_{1}$, then $w_{2}$ is inconsistent with the potential knowledge that $S$ possesses at $w_{1}$.

The Euclidean constraint requires that if $w_{3}$ is consistent with everything that $S$ is in a position to know at $w_{1}$, and $w_{3}$ is not consistent with everything that $S$ is in a position to know at $w_{2}$ then $w_{2}$ is not consistent with everything that $S$ is in a position to know at $w_{1}$. In effect, if the potential knowledge that $S$ possesses at $w_{2}$ is, in some respect, stronger than the potential knowledge that $S$ possesses at $w_{1}$, then $w_{2}$ is inconsistent with the potential knowledge that $S$ possesses at $w_{1}$. If we impose a Euclidean constraint, in addition to a reflexivity constraint, then $R$ will be an equivalence relation - it will serve to partition $W$ into sets of worlds that all stand in relation $R$ to one another, but to no worlds outside of the set. This would appear to capture the idea that we have a kind of privileged 'introspective' access to our own potential knowledge - the worlds that will be consistent with $S$ 's potential knowledge at $w$ will be just those at which $S$ possesses exactly the same potential knowledge.

Kripke semantics, so understood, provides us with an additional resource when it comes to evaluating the plausibility of the eight optional principles. Provided one takes the Kripke-semantic clause for $K$ to be appropriate, the plausibility or otherwise of any of these principles will be tethered to the plausibility of the corresponding constraint which may, in some cases, be easier to evaluate. This, surely, is one of the most tangible benefits of an adequate semantic framework - it can provide us with an independent 'entry point' into evaluating the plausibility of various logics. Our primary concern here, of course, is not with the plausibility of any of the optional principles but, rather, with the plausibility of $(\mathrm{K})$ itself. And, when it comes to this question, the Kripke semantic framework effectively falls silent-whatever semantic assumptions $(\mathrm{K})$ embodies are, in some way, smuggled into the very framework itself. 


\section{Generalising Kripke semantics}

In order to understand the semantic assumptions underlying $(\mathrm{K})$, we require a more general framework - a framework relative to which (K) itself stands as an optional principle. One such framework is provided by what is sometimes called minimal or neighbourhood semantics. ${ }^{8}$ Let a neighbourhood frame $\mathcal{F}$ be a pair $\langle W, N\rangle$ where $W$, as before, is a nonempty set of possible worlds and $N$ is a function assigning to each $w \in W$ a set of sets of elements of $W$. The elements of $N(w)$ are sometimes termed the 'neighbourhoods' around w. A neighbourhood model $\mathcal{M}$ based upon the frame $\langle W, N\rangle$ is a triple $\langle W, N, I\rangle$ where $I$, as before, is a mapping from non-negative integers to subsets of $W$. What it is for a sentence $\phi$ to be true at a world $w$ in a neighbourhood model $\left(\models_{w}^{\mathcal{M}} \phi\right)$ is defined by recycling the recursive clauses listed above, with the exception of the clause for ' $K$ ' which now reads:

$$
\vDash_{w}^{\mathcal{M}} K \phi \text { iff }\left\{w \in W: \models_{w}^{\mathcal{M}} \phi\right\} \in N(w)
$$

According to this clause, $K \phi$ is true at a world $w$ in a neighbourhood model iff the set of worlds at which $\phi$ is true is one of the neighbourhoods around $w$.

A sentence $\phi$ might be described as valid in a neighbourhood model $\mathcal{M}=$ $\langle W, N, I\rangle$ just in case for every $w \in W \models_{w}^{\mathcal{M}} \phi$. In this case we write $\vDash^{\mathcal{M}} \phi$. A sentence $\phi$ might be described as valid in a neighbourhood frame $\mathcal{F}=\langle W, N\rangle$ just in case it is valid relative to any model based on that frame. In this case we write $\models^{\mathcal{F}} \phi$. Finally, a sentence might be described as valid relative to a class of neighbourhood frames just in case it is valid with respect to every frame in the class.

SPC does not preserve validity in all neighbourhood models. Consider a neighbourhood model $\mathcal{M}=\langle W, N, I\rangle$. Suppose $W$ consists of just two worlds $w_{1}$ and $w_{2}$ such that $N\left(w_{1}\right)=\left\{\left\{w_{1}\right\},\left\{w_{2}\right\}\right\}$. Suppose that the assignment ensures that $P_{0}$ is true and $P_{1}$ is true at $w_{1}$, and $P_{0}$ false and $P_{1}$ true at $w_{2} . P_{0} \supset P_{1}$ is valid in $\mathcal{M}$. Given the new clause for $K$, however, it follows that $K P_{0}$ is true at $w_{1}$ while $K P_{1}$ is not.

Say that a neighbourhood frame is supplemented iff the set of neighbourhoods around a world is closed under the superset relation - that is, a neighbourhood frame $\mathcal{F}=\langle W, N\rangle$ is supplemented iff $\forall x \in W, \forall \Pi \in N(x), \Pi \subseteq \Delta \rightarrow \Delta \in N(x)$. SPC does preserve validity in any supplemented neighbourhood frame. Suppose $\phi \supset \psi$ is valid in a supplemented neighbourhood model $\mathcal{M}=\langle W, N, I\rangle$, and that $K \phi$ holds at some world $w \in W$. It follows, given the clause for $K$, that the set of worlds $\Pi$ at which $\phi$ is true is an element of $N(w)$. Since $\phi \supset \psi$ holds at every world in $W$, the set of worlds $\Delta$ at which $\psi$ is true must be a superset of $\Pi$. Since $\mathcal{M}$ is a supplemented model, $\Delta$ must be an element of $N(w)$ in which case, given the semantic clause for $K, K \psi$ is true at $w$. Thus, $K \phi \supset K \psi$ holds at every world in $W$ and is valid in $\mathcal{M}$.

$(\mathrm{K})$, however, is not valid in all supplemented neighbourhood frames. Consider a neighbourhood model $\mathcal{M}=\langle W, N, I\rangle$. Suppose $W$ consists of just two worlds $w_{1}$ and $w_{2}$ such that $N\left(w_{1}\right)=\left\{\left\{w_{1}\right\},\left\{w_{2}\right\}, W\right\}$. $\mathcal{M}$ clearly meets the conditions for a supplemented neighbourhood model. Suppose the valuation ensures that $P_{0}$ is true and $P_{1}$ false at $w_{1}$ and $P_{0}$ and $P_{1}$ are both false at $w_{2}$. Given the recursive clause for $K$, it follows straightforwardly that $K\left(P_{0} \supset P_{1}\right)$ and $K P_{0}$ hold at $w_{1}$ but $K P_{1}$ does

\footnotetext{
${ }_{8}^{8}$ For a detailed discussion of this framework, see Chellas (1980, Ch. 7) or Pacuit (2017).
} 
not. For a simple countermodel to (A) suppose that $P_{0}$ is true and $P_{1}$ is false at $w_{1}$ and $P_{1}$ is true and $P_{0}$ is false at $w_{2}$. In this case $K\left(P_{0} \wedge P_{1}\right)$ will be false at $w_{1}$ while $K\left(P_{0}\right)$ and $K\left(P_{1}\right)$ will both be true. This example also serves to show that MPC does not preserve validity in all supplemented neighbourhood frames.

Say that a neighbourhood frame $\mathcal{F}=\langle W, N\rangle$ satisfies closure under (finite) intersections iff $\forall x \in W, \forall \Pi, \Delta \in N(x), \Pi \cap \Delta \in N(x)$. (A) is valid in any neighbourhood frame that satisfies that condition. Consider a neighbourhood model $\mathcal{M}=\langle W, N, I\rangle$ that satisfies closure under intersections. Suppose that $K \phi$ and $K \psi$ are true at some world $w \in W$. It follows, given the recursive clause for $K$, that the set of worlds $\Pi$ at which $\phi$ is true is an element of $N(w)$ and the set of worlds $\Delta$ at which $\psi$ is true is an element of $N(w)$. Since $\mathcal{M}$ satisfies closure under intersections, $\Pi \cap \Delta$ must be an element of $N(w)$. Since $\Pi \cap \Delta$ is the set of worlds at which $\phi \wedge \psi$ is true, it follows, given the semantic clause for $K$, that $K(\phi \wedge \psi)$ is true at $w$. Since (A) is valid in any neighbourhood frame that satisfies closure under intersections and SPC preserves validity in any supplemented neighbourhood frame, it follows that $(\mathrm{K})$ is valid in all supplemented neighbourhood frames that satisfy closure under intersections.

Say that a neighbourhood frame $\mathcal{F}=\langle W, N\rangle$ satisfies full closure under intersections iff $\forall x \in W, \forall \Pi \subseteq N(x), \bigcap \Pi \in N(w)$. Closure under finite intersections guarantees full closure under intersections provided that $N(w)$ is non-empty and finite for any $w \in W$. Supplemented neighbourhood frames that satisfy full closure under intersections are, in effect, thinly veiled Kripke frames. If $N(w)$ is fully closed under intersections and closed under the superset relation then $\bigcap N(w)$ will be a member of $N(w)$ and, furthermore, $\Pi \in N(w)$ iff $\Pi \supseteq \bigcap N(w)$. The neighbourhood-semantic clause for $K$ could then be rewritten:

$$
\vDash_{w}^{\mathcal{M}} K \phi \text { iff }\left\{w \in W: \models_{w}^{\mathcal{M}} \phi\right\} \supseteq \bigcap N(w)
$$

Finally, if we define a binary relation $R$ as follows: $w_{1} R w_{2}$ iff $w_{2} \in \bigcap N\left(w_{1}\right)$, we are back to the Kripke-semantic clause for $K$.

One complaint that is sometimes made against neighbourhood semantics is that, in constrast to Kripke semantics, it strikes one as almost empty or contrived (Sylvan 1989). The complaint is difficult to make precise, but also difficult to completely ignore, particularly if the function $N$ is left unexplained. With $N$ unexplained, neighbourhood semantics may provide a way of modelling a range of weak epistemic logics-but it would not provide any independent way of evaluating the plausibility of such logics. In the present setting, though, a more substantial interpretation of $N$ may be available.

As discussed in Sect. 1, one powerful motivation for rejecting multiple premise closure for knowledge springs from the idea that knowledge should require only the minimisation, and not the complete elimination, of falsity risk. To make sense of this idea, we need some notion of epistemic probability on which being in a position to know something is compatible with its negation having an epistemic probability that is greater than $0 .{ }^{9}$ Let $\operatorname{Pr}_{w}$ be a probability function, defined over a field $F_{w}$ of subsets of

\footnotetext{
${ }^{9}$ In an infinite probability space a proposition may have a probability of 1 (and its negation a probability of 0 ), even though it is false at some worlds in the space. We can imagine a view on which being in a position to know something requires that its negation have an epistemic probability of 0 , but is compatible with its negation being true in some epistemically possible worlds. This could be classed as a kind of 'risk minimisation' view, albeit one which won't pose a threat to multiple premise closure.
} 
$W$-that is, a set of subsets of $W$ that includes $W$ itself and is closed under intersection and complementation in $W$. If $\Pi$ is a set in $F_{w}$, let $P r_{w}(\Pi)$ be taken to represent the epistemic probability, for $S$ at $w$, that she is located at one of the worlds in $\Pi$.

With this machinery in place, $N(w)$ might be interpreted as the set of sets of worlds in $F_{w}$ that are reckoned to be sufficiently probable by $\operatorname{Pr}_{w}: N(w)=\left\{\Pi \in F_{w}\right.$ : $\left.\operatorname{Pr}_{w}(\Pi)>t \& C(\Pi)\right\}$, where $t$ is a threshold close to, but less than, 1, and $C$ is a placeholder for any further constraints that we wish the members of $N(w)$ satisfy. On this interpretation, what the neighbourhood semantic clause for $K$ says is that $S$ is in a position to know a sentence $\phi$ at a world $w$ only if the set of worlds at which $\phi$ is true satisfies condition $\mathrm{C}$ and has an epistemic probability, for $S$ at $w$, that exceeds a threshold $t$, for some $t$ that is close to but less than 1. As such, the semantic clause would appear to capture the central claim of the risk-minimisation theory-namely, that being in a position to know something requires the minimisation of falsity risk (Kyburg and Teng 2002). Other requirements on knowledge can be captured by the free constraint $C$. If, for instance, knowledge is required to be factive so that one can only know truths, then we should require that $N(w)$ only include sets that contain $w$. If we were modelling a logic of justification, rather than knowledge, no such constraint would not be needed, and it may be possible to interpret $N(w)$ as including all sufficiently probable sets of worlds, allowing $C$ to lapse altogether. Even if we impose no additional constraints, this semantic framework can give rise to several different logics for the $K$-operators, depending on where we set the threshold $t .{ }^{10}$ All of these logics will be stronger than the weak logic that we call EMN, following (Chellas 1980), and that we will formally define at the beginning of Sect. . $^{11}$

Notice that if $\operatorname{Pr}_{w}(\Pi)>t$ and $\Pi \subseteq \Delta$, it follows that $\operatorname{Pr}_{w}(\Delta)>t$. As such, $N(w)$, on the present interpretation, will be closed under the superset relation, on the assumption that any further constraint $C$ is similarly closed. Unless tempted by further closure-violating conditions upon knowledge, risk minimisation theorists who accept the present interpretation of $N$ should countenance only supplemented neighbourhood frames. This is as it should be-as discussed in the last section, risk minimisation theorists have no motivation to reject SPC. As noted above, if we relax the supplementation requirement, neighbourhood semantics can be used to model epistemic logics in which even SPC fails. Perhaps it is possible to interpret unsupplemented neighbourhood frames in a way that comports with the anti-sceptical motivation for rejecting SPC — but we won't explore this here.

\footnotetext{
10 If the threshold $t$ is set at 0.5 or higher then any two members of $N(w)$ must overlap (have a nonempty intersection) - a constraint which will validate (D). Higher values for $t$ will yield further constraintsif, for instance, $t$ is set at $\frac{2}{3}$ or above, it will turn out that any three members of $N(w)$ must have a nonempty intersection. In general, for any $n$, the constraint that any $n$ members of $N(w)$ must have a nonempty intersection will be yielded by a threshold of $\frac{n-1}{n}$ or higher. All such constraints will of course be guaranteed by the (T)-validating constraint that every member of $N(w)$ contain w-and every one of these constraints will serve to validate a principle intermediate in strength between (D) and (T). We won't pursue this further here.

11 Kyburg and Teng (2002) may be read as endorsing EMN as a logic for a knowledge operator, appealing to a kind of unadorned risk minimisation conception of knowledge. Despite the way Kyburg and Teng present things, the failure of factivity makes it difficult to take EMN seriously as a logic of knowledge. It may have more promise as a logic of justification but, partly for reasons explored in the previous footnote, is arguably too weak even for this purpose.
} 
On the present interpretation, $N(w)$ will not, in general, be closed under intersections- $\operatorname{Pr}_{w}(\Pi)>t$ and $\operatorname{Pr}_{w}(\Delta)>t$ are compatible with $\operatorname{Pr}_{w}(\Pi \cap \Delta) \leq t$, for any $t<1$. The requirement that $N(w)$ be closed under intersections should be understood, on the present interpretation, as the requirement that $t$ be set at 1 . As discussed, it is this requirement that will, in effect, take us back into the realm of Kripke semantics, normal epistemic logics and MPC. Kripke semantics can be portrayed, then, as a limiting case of the present semantics, applicable when knowledge requires epistemic certainty-the complete elimination, and not merely the minimisation, of error risk.

Neighbourhood semantics seems a good fit with a risk minimisation picture of knowledge - there is something inherent within the risk minimisation picture that $\mathrm{N}$ could be taken to represent. ${ }^{12}$ But for those who are motivated to abandon MPC for reasons of compartmentalisation, rather than reasons of risk minimisation, neighbourhood semantics seems a less natural choice. There is nothing, or nothing obvious, within the compartmentalisation picture, that the function $\mathrm{N}$ could be taken to represent. While neighbourhood semantics can be used to model the weak epistemic logics to which a compartmentalisation theorist may be attracted, such a theorist should not regard it as much more than a convenient technical device for doing so.

For those attracted to the compartmentalisation picture, we suggest an alternative semantic approach that is much less familiar but is mentioned in Chellas (1980, pp. 74-75) and developed further in Milne (1993). ${ }^{13}$ As it lacks a standard name, we term it plurality semantics. Let a plurality frame $\mathcal{F}$ be a pair $\langle W, \mathcal{R}\rangle$, where $W$ is, as always, a nonempty set of possible worlds and $\mathcal{R}$ is a non-empty set of binary relations on W. A plurality model $\mathcal{M}$ based upon the frame $\langle W, \mathcal{R}\rangle$ is a triple $\langle W, \mathcal{R}, I\rangle$, where $I$, as before, is a mapping from nonnegative integers to subsets of $W$. Truth at a world in a plurality model $\left(\models_{w}^{\mathcal{M}} \phi\right)$ is defined using the same recursive clauses for the extensional operators and the following modification of the Kripke-semantic clause for $K$ :

$$
\vDash_{w}^{\mathcal{M}} K \phi \text { iff } \exists R \in \mathcal{R}, \quad \forall x \in W, \quad w R x \rightarrow \vDash_{x}^{\mathcal{M}} \phi
$$

Acording to this clause, $K \phi$ is true at a world $w$ in a plurality model iff there is a relation $R$ in $\mathcal{R}$ that connects $w$ only to worlds at which $\phi$ is true. ${ }^{14}$

A sentence $\phi$ might be described as valid relative to a plurality model $\mathcal{M}=$ $\langle W, \mathcal{R}, I\rangle$ just in case for every $w \in W \models_{w}^{\mathcal{M}} \phi$. In this case we write $\models^{\mathcal{M}} \phi$. A sentence $\phi$ might be described as valid relative to a plurality frame $\mathcal{F}=\langle W, \mathcal{R}\rangle$ just in case it is valid relative to every model based on that frame. In this case we write

\footnotetext{
12 Williamson (2015) discusses epistemic logics for knowledge and for justified belief in which multiple premise closure fails, pointing out the connection with a risk minimisation conception of such notions. He notes that these logics can be modelled using neighbourhood semantics, but does not discuss how the semantics should be interpreted in the present setting.

13 A semantic framework that is motivated by similar considerations, but differs in the implementation, is presented in Schotch and Jennings (1981a,b). See also the discussion of multi-relational frames in Humberstone (2015, pp. 245-47). This semantic framework also bears a close relation to that set out in Fagin and Halpern (1987, Sect. 6). While plurality semantics works with relations, theirs is a modification of neighbourhood semantics that works, in effect, with the sets of worlds accessible from those relations.

14 If there are finitely many relations $\left\{R_{1}, \ldots, R_{n}\right\}$ in $\mathcal{R}$, and if there are corresponding normal operators $K_{i}$ in the object language, $K \phi$ can be equivalently defined as $K_{1} \phi \vee \cdots \vee K_{n} \phi$.
} 
$\models^{\mathcal{F}} \phi$. Finally, a sentence might be described as valid relative to a class of plurality frames just in case it is valid with respect to every frame in the class.

That $(\mathrm{K})$ is not valid in all plurality frames is easily demonstrated. Consider a plurality model $\mathcal{M}=\langle W, \mathcal{R}, I\rangle$. Suppose $W$ consists of just three worlds $w_{1}, w_{2}$ and $w_{3}$ and $\mathcal{R}$ contains just two relations $R$ and $R^{\prime}$ with just the following instances: $w_{1} R w_{2}$ and $w_{1} R^{\prime} w_{3}$. Suppose finally that the valuation ensures that $P_{0}$ is true and $P_{1}$ is false at $w_{2}$ and $P_{0}$ and $P_{1}$ are both false at $w_{3}$. Given the new recursive clause for $K$ it follows straightforwardly that $K\left(P_{0} \supset P_{1}\right)$ and $K P_{0}$ hold at $w_{1}$ but $K P_{1}$ does not. For a simple countermodel to (A) suppose that the assignment ensures that $P_{0}$ is true and $P_{1}$ is false at $w_{2}$ and $P_{1}$ is true and $P_{0}$ is false at $w_{3}$. In this case $K\left(P_{0} \wedge P_{1}\right)$ will be false at $w_{1}$ while $K\left(P_{0}\right)$ and $K\left(P_{1}\right)$ will both be true. MPC does not preserve validity in every plurality frame.

SPC, however, will preserve validity in every plurality frame. Suppose $\phi \supset \psi$ is valid in a plurality model $\mathcal{M}=\langle W, \mathcal{R}, I\rangle$. Suppose that $K \phi$ holds at some world $w \in W$. It follows, given the recursive clause for $K$, that there is some relation $R \in \mathcal{R}$ such that $\phi$ is true at every world to which $w$ is connected via $R$. Since $\phi \supset \psi$ holds at every world in $W$ it follows, further, that $\psi$ is true at every world that $w$ accesses via $R$ in which case, given the semantic clause for $K, K \psi$ is true at $w$. Thus, $K \phi \supset K \psi$ holds at every world in $W$ and is valid in $\langle W, \mathcal{R}, I\rangle$.

Likewise, KG will preserve validity in every plurality frame. Suppose $\phi$ is valid in a plurality model $\mathcal{M}=\langle W, \mathcal{R}, I\rangle$, and pick $w \in W$. Since $\mathcal{R}$ is non-empty, there is $R \in \mathcal{R}$. Since $\phi$ is true at every world in the model, it is true at every world that $w$ accesses via $R$. Hence, given the semantic clause for $K, K \phi$ is true at $w$. Since $w$ was arbitrary, $K \phi$ is valid.

Kripke models can be portrayed as a subclass of plurality models-namely, those in which $\mathcal{R}$ contains but a single relation. In this case, the plurality-semantic clause for $K$ will simply turn out to be a verbose rephrasing of the Kripke-semantic clause- the two will clearly output the same values when $\mathcal{R}=\{R\}$. Call the following constraint uniqueness:

$$
\exists R \in \mathcal{R}, \quad \forall R^{\prime} \in \mathcal{R}, \quad R=R^{\prime}
$$

Uniqueness is the constraint that reduces a plurality model, in effect, to a Kripke model. As such, uniqueness is a semantic constraint that guarantees the validity of (A) and (K) within plurality semantics. As will be demonstrated, it is not the weakest semantic constraint to do so.

In the Kripke semantics for epistemic logic, the relation $R$ was understood as encoding information about consistency with an agent's potential knowledge at various possible worlds- what it means for world $w_{1}$ to stand in relation $R$ to world $w_{2}$ is that $w_{2}$ is consistent with what $S$ is in a position to know at $w_{1}$. On the compartmentalisation picture, the intended interpretation of the relations in $\mathcal{R}$ seems clear enough-each will encode information about consistency with the potential knowledge of a particular compartment at various possible worlds. Recall that compartments can be interpreted, at a minimum, as repositories of potential knowledge. On this interpretation, what the plurality-semantic clause for $K$ says is that $S$ is in a position to know a sentence $\phi$ at a world $w$ iff at least one of $S$ 's underlying compartments is such that $\phi$ is true at every world that is consistent with what it is in a position to know at $w$. In this case, the 
plurality-semantic clause for $K$ captures the central claim of the compartmentalisation picture-that an individual counts as being in a position to know something iff one of his underlying compartments does. If we are dealing with the knowledge of a group, rather than a compartmentalised individual, then each of the relations in $\mathcal{R}$ will encode information about consistency with the potential knowledge of a given member at various possible worlds, and the plurality-semantic clause for $K$ will capture the claim that a group counts as being in a position to know something whenever one of its members does.

The requirement that $\mathcal{R}$ contain but a single relation should be understood, on the present interpretation, as the requirement that an agent have only one underlying compartment or a group have only one member. This, as discussed, is a requirement that will take us back into the realm of Kripke semantics and normal epistemic logics. Kripke semantics can be portrayed, then, as a limiting case of the present semantics, applicable to non-compartmentalised agents and exclusive groups.

\section{Comparing neighbourhood semantics and plurality semantics}

We have seen that Kripke semantics can be portrayed as a limiting case of either neighbourhood semantics or plurality semantics. It remains to clarify the relationship between the latter two.

There is a good sense in which neighbourhood semantics is more general. If a sentence is valid in every neighbourhood frame, it is valid in every plurality frame, as will be shown below. However, the converse does not hold: $K \phi \supset K(\phi \vee \psi)$ is valid in every plurality frame, but not in every neighbourhood frame. Furthermore, $K \top$ is not valid in a neighbourhood frame in which $N(w)$ is empty for some $w$, but our requirement that $\mathcal{R}$ be non-empty ensures that it is valid in every plurality frame.

There is, however, a certain correspondence between the class of plurality frames and the class of supplemented neighbourhood frames that contain the unit, i.e. are such that $W \in N(w)$ for all $w \in W$. These two classes are equivalent in the sense that exactly the same sentences of $\mathcal{L}$ are valid in them. In fact, there is an even tighter correspondence. Say that $\mathcal{F}=\langle W, N\rangle$ and $\mathcal{F}^{\prime}=\left\langle W^{\prime}, \mathcal{R}\right\rangle$ are point-wise equivalent if $W=W^{\prime}$ and for all valuations $I, \models_{w}^{\mathcal{M}} \phi$ iff $\models_{w}^{\mathcal{M}^{\prime}} \phi$ for all $\phi \in \mathcal{L}$ and all $w \in W$ (where $\mathcal{M}=\langle W, N, I\rangle$ and $\mathcal{M}^{\prime}=\langle W, \mathcal{R}, I\rangle$ ).

Let ()$^{*}$ be a function that maps a plurality frame $\mathcal{F}=\langle W, \mathcal{R}\rangle$ to the neighbourhood frame defined as follows:

$$
\mathcal{F}^{*}=\langle W, N\rangle, \text { with } N(w):=\{X: \exists R \in \mathcal{R}(\forall v(w R v \rightarrow v \in X))\}
$$

We can then show the following:

Theorem 4.1 For every plurality frame $\mathcal{F}, \mathcal{F}^{*}$ is supplemented, contains the unit, and is point-wise equivalent to $\mathcal{F}$.

Proof Suppose that $X \in N(w)$, and that $X \subseteq Y$. There is $R \in \mathcal{R}$ such that $v \in X$ whenever $w R v$. Since $X \subseteq Y, v \in Y$ whenever $w R v$. Hence $Y \in N(w)$, which shows that $\mathcal{F}^{*}$ is supplemented. Since $\mathcal{R}$ is non-empty, there is $R$ such that $v \in W$ 
whenever $w R v$. Hence $W \in N(w)$, and $\mathcal{F}^{*}$ contains the unit. Given a valuation, a straightforward induction on the complexity of $\mathcal{L}$-sentences shows that truth-values in the resulting models agree.

For the converse result, define a function ()$^{+}$that maps a neighbourhood frame $\mathcal{F}$ $=\langle W, N\rangle$ to the following plurality frame: ${ }^{15}$

$$
\mathcal{F}^{+}=\left\langle W,\left\{R_{X}: \exists w \in W(X \in N(w))\right\}\right\rangle,
$$

where the relation $R_{X}$ relates $w$ to each world in $R_{X}(w)$, defined by

$$
R_{X}(w)= \begin{cases}X & \text { if } X \in N(w) \\ W & \text { otherwise }\end{cases}
$$

Theorem 4.2 For every supplemented neighbourhood frame $\mathcal{F}$ that contains the unit, $\mathcal{F}^{+}$is a point-wise equivalent plurality frame.

Proof We fix a valuation $I$ to obtain models $\mathcal{M}$ and $\mathcal{M}^{+}$. Define $[\phi]=\left\{x: \models_{x}^{\mathcal{M}} \phi\right\}$. We use induction on the complexity of formulas.

Suppose $\models_{w}^{\mathcal{M}} \phi$. The argument is straightforward for the cases where $\phi$ is atomic, or a negation, or a conjunction. So assume $\models_{w}^{\mathcal{M}} K \psi$. Then $[\psi] \in N(w)$, and hence $R_{[\psi]} \in \mathcal{R}^{+}$and $R_{[\psi]}(w)=[\psi]$. Pick $v$ such that $w R_{[\psi]} v$. Then $v \in[\psi]$, and by the induction hypothesis, $\models_{v}^{\mathcal{M}^{+}} \psi$, and hence $\models_{w}^{\mathcal{M}^{+}} K \psi$.

For the other direction, suppose $\models_{w}^{\mathcal{M}^{+}} \phi$. We can again focus on the case where $\phi=K \psi$. Then for some $v$ and some $X$ with $X \in N(v), \models_{u}^{\mathcal{M}^{+}} \psi$ for every $u \in R_{X}(w)$. Fix such $v$ and $X$. Case (i): $X \in N(w)$. Then $R_{X}(w)=X$, and by the induction hypothesis, $\models_{u}^{\mathcal{M}} \psi$ for every $u \in X$. Since $\mathcal{M}$ is supplemented, $\models_{w}^{\mathcal{M}} K \psi$. Case (ii): $X \notin N(w)$. Then $R_{X}(w)=W$. By the induction hypothesis, $\models_{u}^{\mathcal{M}} \psi$ for every $u \in W$. Since $\mathcal{M}$ contains the unit, $W \in N(w)$, and hence $\models_{w}^{\mathcal{M}} K \psi$.

In general, there will be more than one plurality frame that is point-wise equivalent to a given supplemented neighbourhood frame that contains the unit. The construction used above only gives one example. We may note that adding relations that are weaker than others already in a plurality frame produces a point-wise equivalent framewhere a relation is weaker than another iff the obtaining by the former is entailed by the obtaining of the latter. More precisely, if for every world $w$, there is a relation $R^{\prime} \in \mathcal{R}$ such that $w R v$ whenever $w R^{\prime} v$, then $\langle W, \mathcal{R} \cup\{R\}\rangle$ is pointwise equivalent to $\langle W, \mathcal{R}\rangle$. This is a consequence of the evaluation clause for $K$ : adding further relations never falsifies a true claim of the form $K \phi$, while adding a weaker relation never verifies a claim of the form $K \phi$ that was not already verified by another relation.

There is thus a sense in which plurality frames have surplus structure: they have more structure than is needed to evaluate the formulas of our language $\mathcal{L}$. In this respect, neighbourhood semantics is superior. On the other hand, plurality semantics is, in our view, more natural and intuitive, especially for those of us who are accustomed to

\footnotetext{
$15 \mathrm{We}$ are grateful to an anonymous referee for suggesting this definition.
} 
thinking of modal operators in terms of accessibility relations and of modal principles in terms of structural constraints upon them.

As a consequence of the correspondence between plurality models and supplemented neighbourhood models that contain the unit, many technical results about plurality semantics can be obtained as corollaries to results about neighbourhood semantics.

Definition 4.1 Condition $C^{\prime}$ on plurality frames is a pluralisation of condition $C$ on neighbourhood frames just in case for every plurality frame $\mathcal{F}, \mathcal{F}^{*}$ satisfies condition $C$ iff $\mathcal{F}$ satisfies $C^{\prime}$.

If $C^{\prime}$ is a trivial condition on a plurality frame-being satisfied by all of them - then $C^{\prime}$ is a pluralisation of the condition of both being supplemented and containing the unit.

Theorem 4.3 If system $S$ is determined by the class of neighbourhood frames satisfying condition $C$, and $C^{\prime}$ is a pluralisation of $C$, then $S$ is also determined by the class of plurality frames satisfying $C^{\prime}$.

Proof Let $\mathcal{F}$ be a plurality frame satisfying $C^{\prime}$. Given that $C^{\prime}$ is a pluralisation of $C$, $\mathcal{F}^{*}$ satisfies $C$. By the soundness result for $C$-frames, every theorem of $S$ is valid in $\mathcal{F}^{*}$. Since $\mathcal{F}$ and $\mathcal{F}^{*}$ are point-wise equivalent (Theorem 4.1), every theorem of $S$ is valid in $\mathcal{F}$. Hence $S$ is sound for frames satisfying condition $C^{\prime}$.

Suppose now that $\phi$ is not a theorem of $S$. By the completeness result for $C$-frames, $\phi$ fails in some model $\mathcal{N}$ based on a frame $\mathcal{F}^{\prime}$ satisfying $C$. Then there is a plurality frame $\mathcal{F}$ such that $\mathcal{F}^{*}=\mathcal{F}^{\prime}$, namely, $\mathcal{F}=\mathcal{F}^{\prime+}$. (It is easily verified that in general, $\mathcal{F}^{+*}=\mathcal{F}$.) Since the frames are point-wise equivalent, $\phi$ fails in model $\mathcal{M}$ based on $\mathcal{F}$, with the same valuation as $\mathcal{N}$. Since $\mathcal{F}$ satisfies $C^{\prime}, S$ is complete for the class of plurality frames satisfying $C^{\prime}$.

Before we can exploit this result, some book-keeping is needed. We noted in Sect. 2 that a $\mathbf{K}$-logic could be defined as a set of $\mathcal{L}$-sentences that includes all truth functional tautologies and admits the rules MP, KG and MPC. Let a EMN-logic be a set of $\mathcal{L}$-sentences that includes all truth-functional tautologies, and admits the rules MP, KG, and SPC. The minimal EMN-logic is simply called EMN. We shall use $\mathbf{E M N} X_{1} \ldots X_{n}$ to refer to the smallest EMN-logic that includes all instances of the principles $X_{1}, \ldots, X_{n}$, taken from the list given in Sect. 2 . The symbol $S$ is used as a variable ranging over EMN-logics. As usual, the turnstile symbolizes theoremhood: $\vdash_{S} \phi$ iff $\phi \in S$.

The system EMN is determined by the class of supplemented neighbourhood frames that contain the unit [Chellas (1980, pp. 258-259)]. Given Theorem 4.3 and the above observation that the trivial condition is a pluralisation of the condition of being supplemented and containing the unit, we get:

Theorem 4.4 EMN is determined by the class of all plurality frames.

We can also prove this result directly, without relying on the textbook result about neighbourhood models. Doing so will pay dividends later in the paper, when we prove determination results with respect to certain classes of plurality frames. 
It is routine to verify that $\mathbf{E M N}$ is sound with respect to the class of all plurality frames. For the completeness part, we use a construction of canonical models devised by Milne (1993) that will be useful for further results too.

For a system $S$, say that a set of sentences $\Gamma \subseteq \mathcal{L}$ is $S$-consistent if there are no $\phi_{1}, \ldots \phi_{n} \in \Gamma$ such that $\vdash_{S} \sim\left(\phi_{1} \wedge \cdots \wedge \phi_{n}\right)$. A set of sentences is $S$-maximal if it is $S$-consistent and has no $S$-consistent proper extensions. According to Lindenbaum's Lemma - a standard result that we shall not prove, and appeal to which shall remain tacit-every $S$-consistent sentences belongs to an $S$-maximal set.

Definition 4.2 If $S$ is a EMN-logic, define the canonical model $\mathcal{M}_{S}=\left\langle W_{S}, \mathcal{R}_{S}, I_{S}\right\rangle$ as follows:

1. $W_{S}=\{w: w$ a $S$-maximal set of sentences $\}$.

2. $\mathcal{R}_{S}=\left\{R_{\phi}: \phi \in L\right\}$, where $R_{\phi}=\left\{\langle w, v\rangle: w, v \in W_{S}\right.$ and $K \phi \notin w$ or $\left.\phi \in v\right\}$.

3. $I_{S}(i)=\left\{w: w \in W_{S}\right.$ and $\left.P_{i} \in w\right\}$.

It is clear that $\mathcal{M}_{S}$ so-defined is a plurality model. We shall now prove that the construction has the fundamental feature we expect a canonical model to have: that a sentence is true at a world just in case it is a member of this world:

Theorem 4.5 If $\mathcal{M}=\langle W, \mathcal{R}, P\rangle$ is the canonical model of a $\mathbf{E M N}$-logic $S$, then for every $w \in W, \phi \in w$ iff $\models_{w}^{\mathcal{M}} \phi$.

Proof By induction on the complexity of $\phi$. If $\phi=P_{i}$, then $\phi \in w$ iff $w \in P_{S}(i)$ (by Definition 4.2.3) iff $\models{ }_{w}^{\mathcal{M}} P_{i}$ (by the evaluation clause for atomic sentences).

The induction steps for the usual propositional connectives are straightforward.

Now suppose $K \phi \in w$. Then for every $v$ in $W_{S}, w R_{\phi} v$ iff $\phi \in v$ (by Definition 4.2.2). By the induction hypothesis, $\vDash_{v}^{\mathcal{M}_{S}} \phi$ for every $v$ with $w R_{\phi} v$. By the evaluation clause for $K, \vDash_{w}^{\mathcal{M}} S \phi$.

For the other direction, suppose that $K \phi \notin w$. We need to show that for every $\psi \in \mathcal{L}, \exists v$ with $w R_{\psi} v$ and $\nvdash_{v}^{\mathcal{M}_{S}} \phi$. Fix $\psi \in \mathcal{L}$. Case (i): $K \psi \notin w$. Then $w R_{\psi} v$ for all $v \in W$. Now since $K \phi \notin w, \nvdash_{S} K \phi$, and since $S$ admits $\mathrm{KG}, \nvdash_{S} \phi$. Hence $\neg \phi$ is consistent, and there is a maximal $v \in W_{S}$ with $\neg \phi \in v$. It follows that $\phi \notin v$, and by the induction hypothesis, $\nvdash_{v}^{\mathcal{M}_{S}} \phi$. Case (ii): $K \psi \in w$. Then $w R_{\psi} v$ iff $\psi \in v$. Suppose that $\phi \in w$ for all $v$ with $w R_{\psi} v$. Then there is no maximal set $v$ with $\psi \in v$ and $\phi \notin v$. Hence $\vdash_{S} \psi \supset \phi$. By SPC, $\vdash_{S} K \psi \supset K \phi$. Hence $K \phi \in w$, contradicting the assumption that $K \phi \notin w$. Thus it is not the case that $\phi \in v$ for all $v$ with $w R_{\psi} v$. Hence $w R v$ for some $v$ with $\nvdash_{w}^{\mathcal{M}_{S}} \phi$ for every $R \in \mathcal{R}_{S}$.

For the completeness part of Theorem 4.4, suppose $\nvdash \neg \phi$. Then there is a EMNmaximal set of sentences $w$ such that $\phi \in w$. By Theorem $4.5, \models_{w}^{\mathcal{M}} \phi$ for the canonical model of EMN. Hence $\phi$ is satisfiable in the class of plurality models.

In the rest of the paper, we shall look at various extensions of the base system EMN. Schema (A), introduced earlier, captures the idea that knowledge is closed under conjunction:

$$
(K \phi \wedge K \psi) \supset K(\phi \wedge \psi)
$$


The system EMNA, that is, the smallest EMN-logic that includes all instances of (A), is just the familiar system $\mathbf{K}$, the smallest normal modal logic, which is determined by the class of all Kripke frames. Typically, $\mathbf{K}$ is characterised as the smallest system that admits MP, KG and contains all tautologies and all instances of $(\mathrm{K})$ :

$$
K(\phi \supset \psi) \supset(K \phi \supset K \psi)
$$

Consider frames that satisfy the constraint that we called uniqueness-i.e. that are such that $\mathcal{R}=\{R\}$ for some $R$. Clearly, there is a validity-preserving one-to-one correspondence from such plurality frames to Kripke frames. Since $\mathbf{K}$ is determined by the class of all Kripke frames, it is also determined by the class of plurality frames satisfying the uniquness constraint. However, this leaves open the question whether $\mathbf{K}$ is also determined by a different class of plurality frames.

Say that $\mathcal{F}=\langle W, \mathcal{R}\rangle$ is sub-closed if for all $w \in W$ and $R, R^{\prime} \in \mathcal{R}$, there is $R^{\prime \prime} \in \mathcal{R}$ such that for all $v, w R^{\prime \prime} v$ only if both $w R v$ and $w R^{\prime} v$. If a frame has only finitely many relations in $\mathcal{R}$, it is sub-closed only if there is a "know-it-all" compartment; that is, a compartment that is in a position to know a sentence if any is.

Theorem 4.6 (A) is defined by the class of sub-closed plurality frames.

Proof To show that $(A)$ is valid in every sub-closed plurality frame, suppose that $\models_{w}^{\mathcal{M}} K \phi$ and $\models_{w}^{\mathcal{M}} K \psi$. Then $\exists R, R^{\prime} \in \mathcal{R}$ such that $\models_{v}^{\mathcal{M}} \phi$ for all $v$ with $w R v$ and $\models_{u}^{\mathcal{M}} \psi$ for all $u$ with $w R^{\prime} u$. Since $\mathcal{R}$ is sub-closed, there is $R^{\prime \prime} \in \mathcal{R}$ such that for all $v, w R^{\prime \prime} v$ only if both $w R v$ and $w R^{\prime} v$. Hence both $\models_{v}^{\mathcal{M}} \phi$ and $\models_{v}^{\mathcal{M}} \psi$ for all $v$ with $w R^{\prime \prime} v$, and hence $\models_{w}^{\mathcal{M}} K(\phi \wedge \psi)$. It follows that $\models_{w}^{\mathcal{M}}(K \phi \wedge K \psi) \supset K(\phi \wedge \psi)$.

It remains to show that every plurality frame that is not sub-closed is the basis of a model in which (A) fails. Suppose that for some $w, R$, and $R^{\prime}$, there is no $R^{\prime \prime}$ such that $w R^{\prime \prime} v$ only if both $w R v$ and $w R^{\prime} v$. Then there is a model $\mathcal{M}$ on this frame with $I(0)=\{v: w R v\}$, and $I(1)=\left\{v: w R^{\prime} v\right\}$. Then $\models_{w}^{\mathcal{M}} K P_{0}$ and $\vDash_{w}^{\mathcal{M}} K P_{1}$ but $\not \forall_{w}^{\mathcal{M}} K\left(P_{0} \wedge P_{1}\right)$, falsifying an instance of $(\mathrm{A})$.

It follows immediately that EMNA is sound for the class of sub-closed plurality frames. To obtain the matching completeness result, note that a plurality frame that satisfies the uniqueness constraint is trivially sub-closed: for if $\mathcal{R}=\{R\}$, then for all $R^{\prime}, R^{\prime \prime} \in \mathcal{R}, R \cap R^{\prime}=R$, and there is $R^{*} \in \mathcal{R}$ such that $R^{*} \subseteq R-R$ itself. Since $K$ is complete for the class of plurality frames with only one relation, it is a fortiori complete for the class of sub-closed plurality frames. Hence we have proved (modulo the completeness proof relative to Kripke frames, which is not given here):

Theorem 4.7 EMNA is determined by the class of sub-closed plurality frames.

In the following, we shall use some of the terms that characterise relations in an extended sense, to characterise classes of relations. So we shall say that $\mathcal{R}$ is reflexive iff every $R \in \mathcal{R}$ is, and that $\mathcal{R}$ is transitive if every $R \in \mathcal{R}$ is, and likewise for the other terms used in the table in Sect. 2.

Theorem 4.8 EMNT is determined by the class of reflexive plurality frames.

Proof For soundness, suppose that $\models_{w}^{\mathcal{M}} K \phi$. Then there is $R \in \mathcal{R}$ such that $\models_{w^{\prime}}^{\mathcal{M}} \phi$ for all $w^{\prime}$ such that $w R w^{\prime}$. Since $\mathcal{R}$ is reflexive, $w R w$. Hence $\models_{w}^{\mathcal{M}} \phi$ and $\models_{w}^{\mathcal{M}} K \phi \supset \phi$. 
For completeness, we verify that the canonical model is reflexive. That is, we show that for $R_{\phi} \in \mathcal{R}_{\text {EMNT }}$, either $K \phi \notin w$ or $\phi \in w$. Suppose that the former is false. Then $K \phi \in w$. Since $K \phi \supset \phi \in$ EMNT, $K \phi \supset \phi \in w$. Since $w$ is closed under MP, $\phi \in w$.

Abstracting from the deductive base provided by the system EMN, we can also obtain a direct correspondence result between $(\mathrm{T})$ and the condition of reflexivity: $(\mathrm{T})$ defines reflexivity, in the sense that it is valid on all reflexive frames, and invalid on all frames that fail to be reflexive. Validity on all reflexive frames follows from the above soundness proof. If $\mathcal{M}$ is not reflexive, then there are $w, R$ such that $w$ does not bear $R$ to itself. If $I(0)=W \backslash\{w\}$, then $\not \neq_{w}^{\mathcal{M}} K P_{0} \supset P_{0}$.

In the next two sections, we will continue to try to develop an autonomous theory of plurality models, rather than transferring results via Theorem 4.3.

\section{EMN4 and transitivity}

In light of the determination result for EMNT, it is tempting to conjecture that if $\mathbf{K} X_{1} \ldots X_{n}$ is determined by the class of Kripke frames in which $R$ satisfies constraints $k\left(X_{1}\right)$ to $k\left(X_{n}\right)$, then $\mathbf{E M N} X_{1} \ldots X_{n}$ is determined by the class of plurality frames in which every $R \in \mathcal{R}$ satisfies $k\left(X_{1}\right)$ to $k\left(X_{n}\right)$. Or at least, we may suspect this to be true for certain simple $X_{i}$ and natural constraints such as those listed in Sect. 2. As it turns out, however, that conjecture is wrong.

Schema (4), to recall, reads as follows:

$$
K \phi \supset K K \phi
$$

It is straightforward to verify that every theorem of EMN4 is valid in every transitive plurality frame. Given the result of the previous section, it suffices to show that (4) is valid in all such frames. Suppose $\models_{w}^{\mathcal{M}} K \phi$. Then there is $R \in \mathcal{R}$ such that whenever $w R v, \models_{v}^{\mathcal{M}} \phi$. We need to show that there is $R^{\prime} \in \mathcal{R}$ such that for every $v$ with $w R^{\prime} v$, there is $R^{\prime \prime} \in \mathcal{R}$ such that $\models_{u}^{\mathcal{M}} \phi$ whenever $v R^{\prime \prime} u$. Setting $R=R^{\prime}=R^{\prime \prime}$ will do: since $R$ is transitive, $w R v$ and $v R u$ entails $w R u$, and hence $\models_{u}^{\mathcal{M}} \phi$ by our assumption. It follows that $\models_{v}^{\mathcal{M}} K \phi$ and $\models_{w}^{\mathcal{M}} K K \phi$.

So far, so good. But EMN4 is not complete for the class of transitive plurality frames. For that class validates $\left(\mathrm{A}^{*}\right)$, which is not a theorem of EMN4: ${ }^{16}$

$$
\left(\mathrm{A}^{*}\right) \quad(K \phi \wedge K K \phi) \supset K(\phi \wedge K \phi)
$$

All instances of $\left(\mathrm{A}^{*}\right)$ are also instances of $(\mathrm{A})$, with $\psi=K \phi$. We can thus view $\left(A^{*}\right)$ as a special case of closure under conjunction.

To show that $\left(\mathrm{A}^{*}\right)$ is valid on the class of transitive frames, let $\mathcal{R}$ be transitive, and suppose that $\nvdash_{w} K(\phi \wedge K \phi)$, and fix $R \in \mathcal{R}$. Then there is $w^{\prime}$ with $w R w^{\prime}$ such that $\nvdash_{w^{\prime}} \phi \wedge K \phi$. Then $\nvdash_{w^{\prime}} \phi$ or $\nvdash_{w^{\prime}} K \phi$. If the former, there is $w^{\prime}$ such that $w R w^{\prime}$ and $\not \nvdash_{w^{\prime}} \phi$. If the latter, then, there is $w^{\prime \prime}$ such that $w^{\prime} R w^{\prime \prime}$ and $\nvdash_{w^{\prime \prime}} \phi$. Since $R$ is transitive, $w R w^{\prime \prime}$. Hence in either case, $\phi$ is false at some world $R$-accessible from $w$. Since $R$ was chosen arbitrarily, it follows that $\nvdash_{w} K \phi$, and hence $\nvdash_{w} K \phi \wedge K K \phi$.

\footnotetext{
16 This could be proved either by exploiting a completeness result for EMN4 relative to neighbourhood frames, or else by exploiting our Theorem 5.2, below.
} 
We can, however, identify another class of plurality frames that does determine EMN4. First we define a relation between relations:

Definition $5.1 R^{\prime}$ attributes $R$ to $R^{\prime \prime}$ in world $w$ iff whenever $w R^{\prime} v$ and $v R^{\prime \prime} t$, then $w R t$.

Or equivalently in set-theoretic notation: $R^{\prime}$ attributes $R$ to $R^{\prime \prime}$ in world $w$ iff $R^{\prime} \circ R^{\prime \prime}(w) \subseteq R(w)$.

If the prime symbols are ignored, the right-hand side looks like it is saying that $R$ is transitive.

We can motivate the terminology by interpreting $R, R^{\prime}$ and $R^{\prime \prime}$ as encoding knowledge of three compartments. If so, and if $R^{\prime}$ attributes $R$ to $R^{\prime \prime}$ in the above sense, then whenever the $R$ compartment knows something, the $R^{\prime}$ compartment knows that the $R^{\prime \prime}$ compartment knows it.

Theorem 5.1 (4) defines the class of frames in which for every $w$ and $R$, there are $R^{\prime}$ and $R^{\prime \prime}$ such that $R^{\prime}$ attributes $R$ to $R^{\prime \prime}$ in $w$.

Proof Suppose that the frame on which $\mathcal{M}$ is based satisfies the condition, and suppose $\vDash_{w}^{\mathcal{M}} K \phi$. Then there is $R \in \mathcal{R}$ such that $\models_{u}^{\mathcal{M}} \phi$ whenever $w R u$. Pick $R^{\prime}$ and $R^{\prime \prime}$ such that $R^{\prime}$ attributes $R$ to $R^{\prime \prime}$ in $w$, and $v$ such that $w R^{\prime} v$. Then whenever $v R^{\prime \prime} u, w R u$, and thus $\models_{u}^{\mathcal{M}} \phi$. Hence $\models_{v}^{\mathcal{M}} K \phi$ and $\models_{w}^{\mathcal{M}} K K \phi$.

Suppose now that $\mathcal{F}$ does not satisfy the condition. Then for some $w$ and $R$, there are no $R^{\prime}$ and $R^{\prime \prime}$ such that $R^{\prime}$ attributes $R$ to $R^{\prime \prime}$ in $w$. Consider a model $\mathcal{M}$ based on $\mathcal{F}$ with $I(0)=\{v: w R v\}$. Then $\models_{w}^{\mathcal{M}} K P_{0}$. But for any $R^{\prime}$ and $R^{\prime \prime}$, there are $v$ and $u$ such that $w R^{\prime} v$ and $v R^{\prime \prime} u$, but not $w R u$. Then $\nvdash_{u}^{\mathcal{M}} P_{0}$, and hence $\not \nvdash_{w}^{\mathcal{M}} K K P_{0}$.

We shall now define a further condition on plurality frames in terms of attribution:

Definition 5.2 $R^{\prime}$ monitors $R$ in $w$ iff $R^{\prime}$ attributes $R$ to $R$; a frame $\mathcal{F}=\langle W, \mathcal{R}\rangle$ is monitored iff for every $R \in \mathcal{R}$ there is $R^{\prime} \in \mathcal{R}$ that monitors $R$ in every $w \in W$.

In set-theoretic notation: $R^{\prime}$ monitors $R$ in $w$ iff $R^{\prime} \circ R(w) \subseteq R(w)$. Note that every transitive relation monitors itself, and that every transitive plurality frame is thus monitored.

Theorem 5.2 EMN4 is determined by the class of all monitored plurality frames.

Proof Soundness follows from Theorem 5.1. For completeness, it is enough to show that the frame of the canonical model of EMN4 is monitored. Let $R_{\phi} \in \mathcal{R}$, and let $w$ be any world. Suppose $v R_{\phi} t$ but not $w R_{\phi} t$. Then since not $w R_{\phi} t, K \phi \in w$ and $\phi \notin t$.


is closed under MP, $K K \phi \in w$. Hence not $w R_{K \phi} v$. Since $w$ was arbitrary, this shows that $R_{K \phi}$ monitors $R_{\phi}$; and since $R_{\phi}$ was arbitrary, the frame is monitored.

\section{EMNB and symmetry}

In the last section, we saw that requiring all relations in the plurality frames to be transitive guarantees the validity of (4), but is stronger than needed for that purpose-(4) 
does not define that condition — and also validates non-theorems of EMN4. Consider now principle (B):

$$
\sim K \sim K \phi \supset \phi
$$

In Kripke semantics, its corresponding condition is symmetry. But while requiring all relations to be transitive gave us more than (4), in a certain sense, requiring all relations to be symmetrical gives us less than (B) in plurality semantics, as we are about to show.

As it turns out, MPC is an admissible rule in EMNB. This has been shown syntactically (Jennings 1981; Humberstone 2015, pp. 181-182), but in the present context it is instructive to appeal to plurality frames in our argument.

Say that $\mathcal{F}$ is quasi-symmetrical iff for every $w \in W$, there is $R \in \mathcal{R}$ such that for all $R^{\prime} \in \mathcal{R}$ and $v \in W$, if $w R v$ then $v R^{\prime} w$. Less formally: at every world there is a compartment $R$ that only relates $w$ to worlds in which no compartment has any potential knowledge that rules out $w$.

Theorem 6.1 (B) is valid in the class of quasi-symmetrical frames.

Proof Suppose that $\not{ }_{w}^{\mathcal{M}} \phi$. Since $\mathcal{R}$ is quasi-symmetrical, there is $R \in \mathcal{R}$ such that for all $R^{\prime} \in \mathcal{R}$ and $v \in W$, if $w R v$ then $v R^{\prime} w$. Let $v$ be any world such that $w R v$. Then, since $v R^{\prime} w$ for every $R^{\prime} \in \mathcal{R}$, and since $\not \nvdash_{w}^{\mathcal{M}} \phi, \not \nvdash_{v}^{\mathcal{M}} K \phi$. Since $v$ was arbitrary, $\vDash_{w}^{\mathcal{M}} K \sim K \phi$, and hence $\not \nvdash_{w}^{\mathcal{M}} \sim K \sim K \phi$.

We can also show the other half of the definability result:

Theorem 6.2 If a frame is not quasi-symmetrical, (B) is not valid on it.

Proof Suppose that $\mathcal{F}$ is not quasi-symmetrical. Then there is $w \in W$ such that for every $R \in \mathcal{R}$, there is $R^{\prime} \in \mathcal{R}$ and $v \in W$ such that $w R v$ and not $v R^{\prime} w$. Let $\mathcal{M}$ be a model on $\mathcal{F}$ with $I(0)=\{w\}$. Then $\models_{w}^{\mathcal{M}} P_{0}$ and thus $\not \nvdash_{w}^{\mathcal{M}} \sim P_{0}$. Pick such $R \in \mathcal{R}$, and $R^{\prime}$ and $v$. Since not $v R^{\prime} w, \models_{v}^{\mathcal{M}} K \sim P_{0}$ and thus $\not \nvdash_{v}^{\mathcal{M}} \sim K \sim P_{0}$. Since $R$ was arbitrary, $\models_{w}^{\mathcal{M}} \sim K \sim K \sim P_{0}$.

So the class of quasi-symmetrical frames is the largest one with respect to which EMNB is sound. So if EMNB is complete with respect to any class of plurality frames, it is complete with respect to the class of quasi-symmetrical ones. The next theorem shows that it is.

Theorem 6.3 EMNB is complete for the class of quasi-symmetrical plurality frames.

Proof Let $\mathcal{M}=\langle W, \mathcal{R}, I\rangle$ be the canonical model of EMNB. Define $\mathcal{M}^{\prime}=$ $\left\langle W, \mathcal{R}^{\prime}, I\right\rangle$, where $\mathcal{R}^{\prime}=\mathcal{R} \cup\left\{R^{*}\right\}$, where $R^{*}=\{\langle w, v\rangle: \phi \in w$ whenever $K \phi \in v\}$, or equivalently, $\{\langle w, v\rangle: \sim K \sim \phi \in v$ whenever $\phi \in w\}$. We need to show two things: (i) $\mathcal{M}$ and $\mathcal{M}^{\prime}$ are pointwise equivalent. (ii) $\mathcal{M}^{\prime}$ is quasi-symmetrical.

(i) The inductive steps for the propositional connectives are straightforward. Suppose $\models_{w}^{\mathcal{M}^{\prime}} K \phi$. Then there is $R \in \mathcal{R}^{\prime}$ such that $\models_{v}^{\mathcal{M}^{\prime}} \phi$ for all $v$ with $w R v$. If $R \in$ $\mathcal{R}$, then the induction hypothesis gives us $\models_{w}^{\mathcal{M}} K \phi$. So suppose that $R=R^{*}$. Then $\models_{v}^{\mathcal{M}^{\prime}} \phi$ for all $v$ with $w R^{*} v$. By the induction hypothesis, $\phi \in v$ for all 
$v$ with $w R^{*} v$. Then $\{\sim K \sim \psi: \psi \in w\} \cup\{\neg \phi\}$ is EMNB-inconsistent. Hence for some $\psi_{1}, \ldots \psi_{n} \in w, \vdash_{\text {EMNB }}\left(\sim K \sim \psi_{1} \wedge \cdots \wedge \sim K \sim \psi_{n}\right) \supset \phi$. By SPC, $\vdash_{\text {EMNB }} K\left(\sim K \sim \psi_{1} \wedge \cdots \wedge \sim K \sim \psi_{n}\right) \supset K \phi$.

We now show that $K\left(\sim K \sim \psi_{1} \wedge \cdots \wedge \sim K \sim \psi_{n}\right) \in w$. Since by hypothesis, $\psi_{1}, \ldots, \psi_{n} \in w$, it is enough to show that $\vdash_{\text {EMNB }} \psi_{1} \wedge \cdots \wedge \psi_{n} \supset K\left(\sim K \sim \psi_{1} \wedge\right.$ $\left.\cdots \wedge \sim K \sim \psi_{n}\right)$. Clearly, $\vdash_{\text {EMNB }} \psi_{1} \wedge \cdots \wedge \psi_{n} \supset K \sim K \sim\left(\psi_{1} \wedge \cdots \wedge \psi_{n}\right)$. Further, $\sim K \sim\left(\psi_{1} \wedge \cdots \wedge \psi_{n}\right) \supset \sim K \sim \psi_{1} \wedge \cdots \wedge \sim K \sim \psi_{n}$ is a theorem of EMN, and hence of EMNB. Since EMNB admits SPC, $\vdash_{\mathbf{E M N B}} K\left(\sim K \sim\left(\psi_{1} \wedge \cdots \wedge \psi_{n}\right)\right) \supset$ $K\left(\sim K \sim \psi_{1} \wedge \cdots \wedge \sim K \sim \psi_{n}\right)$. By the closure of EMNB under propositionallogical consequence, the desired consequence follows.

Hence $K \phi \in w$. Then $\models_{w}^{\mathcal{M}} K \phi$, by Theorem 4.5.

(ii) Let $w$ be any element of $W$, and suppose that $w R^{*} v$ for some $v \in W$. Let $\phi$ be any sentence of $\mathcal{L}$. If $K \phi \notin v$, then $v R_{\phi} w$. If $K \phi \in v$, then since $w R^{*} v, \phi \in w$, and hence $v R_{\phi} w$. This shows that $v R_{\phi} w$. It remains to show that $v R^{*} w$. Suppose $\phi \notin v$. Then $K \sim K \phi \in v$ by (i). Since $w R^{*} v, \sim K \phi \in w$, and hence $K \phi \notin w$, as desired.

Corollary 1 EMNB is detemined by the class of quasi-symmetrical frames.

We can now show that EMNB admits multi-premise closure, i.e. that $\mathbf{E M N B}=\mathbf{K B}$. Suppose frame $\mathcal{F}=\langle W, \mathcal{R}\rangle$ is quasi-symmetrical, and pick $w \in W$ and $R, R^{\prime} \in \mathcal{R}$. By quasi-symmetry, there is $R_{w} \in \mathcal{R}$ such that for all $v$ and $R^{\prime \prime}$, if $w R_{w} v$ then $v R^{\prime \prime} w$. Pick $v$ such that $w R_{w} v$. By quasi-symmetry again, there is $R_{v} \in \mathcal{R}$ such that for all $u$ and $R^{\prime \prime}$, if $v R_{v} u$ then $u R^{\prime \prime} v$. Then $v R_{v} w$, and hence $w R^{\prime \prime} v$ for every $R^{\prime \prime} \in \mathcal{R}$, in particular $R$ and $R^{\prime}$. This shows that $\mathcal{F}$ is sub-closed. By Theorem 4.6, (A) holds in $\mathcal{F}$; and by Theorem 6.3, (A) is a theorem of EMNB. Our earlier observations then entail that EMNB admits MPC.

However, we can easily construct plurality frames in which every relation is symmetrical, but which are not sub-closed. (A frame with three worlds will do, $\mathcal{R}=\left\{R, R^{\prime}\right\}, R$ symmetrically relating one pair of worlds, and $R^{\prime}$ symmetrically relating another pair of worlds.) We know from Theorem 4.6 that there are models based on such frames where (A) fails. Given the result of the previous paragraph, it follows that (B) fails in some symmetrical plurality frames. ${ }^{17}$

We may also note that $(\mathrm{B})$ is entailed by $(\mathrm{T})$ together with $(5)$ :

$$
\sim K \phi \supset K \sim K \phi
$$

It thus follows that EMNT5 is KT5.

In the absence of (T), (5) does not restore multi-premise closure, however. To verify this, consider a model with just two worlds, one where $P_{0}$ is true $\left(w_{P}\right)$ and one where $P_{0}$ is false $\left(w_{\bar{P}}\right)$. Relation $R$ relates both worlds to $w_{P}$, and no others, and relation $R^{\prime}$ relates both worlds to $w_{\bar{P}}$, and no others; and there are no further relations. Then $K P_{0} \wedge K \sim P_{0} \supset K\left(P_{0} \wedge \sim P_{0}\right)$ fails at both $w_{P}$ and $w_{\bar{P}}$.

However, we can check that every instance of (5) holds in every world in that model. In this model, every proposition is either equivalent to $\top, P_{0}, \neg P_{0}$, or $\perp$. Of the four,

\footnotetext{
17 The variable margin frames that Williamson (1994) uses to model the logic of clarity are in effect symmetrical plurality frames which validate (A) but not (B).
} 
the only one such that $\sim K \phi$ is true at some world is $\perp$; indeed, $\sim K \perp$ is equivalent to $\top$. But the semantics ensures that $K \top$ holds, and hence the consequent of (5) for such an instance.

We may further note that various extensions of EMN4 do not admit MPC. Consider a plurality frame with $W=\{w, v, u\}, \mathcal{R}=\left\{R, R^{\prime}\right\}$, where $R$ is the smallest reflexive and symmetrical relation that holds between $w$ and $v$, and $R^{\prime}$ is the smallest reflexive and symmetrical relation that holds between $w$ and $u$. It can be verified-although we shall not do it here - that every instance of (4), (T), (0.2), (0.3), and (0.4) is valid on that frame. From among the candidate principles listed earlier, only (A), (B), and (5) may fail in models based on that frame. This illustrates that epistemic logics which do not admit MPC may still be quite strong in other respects.

\section{Conclusion}

In this paper we have set out two rather different motivations for rejecting a multiple premise closure principle for knowledge. The first of these appeals to the idea that knowledge requires minimising, but not eliminating, the risk of falsity, while the second appeals to the idea that knowers may be compartmentalised in their thinking. We have described a series of epistemic logics in which closure fails and set out two semantic approaches to these logics—neighbourhood and plurality semanticswhich naturally correspond to the two motivations for rejecting closure. As plurality semantics is the less familiar approach, we have offered a more thorough formal exploration of this framework, investigating its links with neighbourhood and Kripke semantics, and outlining how it can deliver some of the best known principles of epistemic logic.

Open Access This article is distributed under the terms of the Creative Commons Attribution 4.0 International License (http://creativecommons.org/licenses/by/4.0/), which permits unrestricted use, distribution, and reproduction in any medium, provided you give appropriate credit to the original author(s) and the source, provide a link to the Creative Commons license, and indicate if changes were made.

\section{References}

Bird, A. (2014). When is there a group that knows? Distributed cognition, scientific knowledge, and the social epistemic subject. In J. Lackey (Ed.), Essays in collective epistemology (pp. 42-63). Oxford: Oxford University Press.

Brown, B., \& Priest, G. (2004). Chunk and permeate, a paraconsistent inference strategy. part i: Theinfinitesimal calculus. Journal of Philosophical Logic, 33, 379-388.

Chellas, B. F. (1980). Modal logic: an introduction. Cambridge: Cambridge University Press.

Cohen, S. (2002). Basic knowledge and the problem of easy knowledge. Philosophy and Phenomenological Research, 65, 309-329.

Dretske, F. (1970). Epistemic operators. Journal of Philosophy, 67, 1007-1023.

Dretske, F. (2005). The case against closure. In M. Steup \& E. Sosa (Eds.), Contemporary debates in epistemology. Oxford: Blackwell.

Fagin, R., \& Halpern, J. Y. (1987). Belief, awareness, and limited reasoning. Artificial Intelligence, 34(1), 39-76. https://doi.org/10.1016/0004-3702(87)90003-8. 
Fagin, R., Halpern, J. Y., Moses, Y., \& Vardi, M. Y. (1995). Reasoning about knowledge. Cambridge, MA: MIT Press.

Hawthorne, J. (2004). Knowledge and lotteries. Oxford: Oxford University Press.

Hintikka, J. (1962). Knowledge and belief: an introduction to the logic of the two notions. Ithaca, NY: Cornell University Press.

Humberstone, L. (2015). Philosophical applications of modal logic. London: College Publications.

Jennings, R. E. (1981). A note on the axiomatisation of the brouwersche modal logic. Journal of Philosophical Logic, 19, 341-343.

Kvanvig, J. (2006). Closure principles. Philosophy Compass, 1, 256-267.

Kyburg, H., \& Teng, C. (2002). The logic of risky knowledge. Theoretical Notes on Computer Science, 67, 254-262.

Lasonen-Aarnio, M. (2008). Single premise deduction and risk. Philosophical Studies, 141, 157-173.

Lenzen, W. (1978). Recent work in epistemic logic. Acta Philosophica Fennica, 30, 1-219.

Lewis, D. (1982). Logic for equivocators. Noûs, 16, 431-441.

Lewis, D. (1996). Elusive knowledge. Australasian Journal of Philosophy, 74, 549-567.

Milne, P. (1993). Minimal doxastic logic. Notre Dame Journal of Formal Logic, 34, 499-526.

Nozick, R. (1981). Philosophical explanations. Cambridge, MA: Harvard University Press.

Pacuit, E. (2017). Neighborhood semantics for modal logic. Cham: Springer.

Rosenkranz, S. (2018). The structure of justification. Mind, 127, 309-338.

Schotch, P. K., \& Jennings, R. E. (1981a). Non-Kripkean deontic logic. In R. Hilpinen (Ed.), New studies in Deontic logic (pp. 149-164). Dordrecht: D. Reidel Publishing.

Schotch, P. K., \& Jennings, R. E. (1981b). Epistemic logic, skepticism, and non-normal logic. Philosophical Studies, 40, 47-67.

Smith, M. (2013). Two notions of epistemic risk. Erkenntnis, 78, 1069-1079.

Smith, M. (2018). The logic of epistemic justification. Synthese, 195, 3857-3875.

Smithies, D. (2012). Mentalism and epistemic transparency. Australasian Journal of Philosophy, 90, 723741.

Spectre, L. (2018). Compartmentalized knowledge. Philosophical Studies. https://doi.org/10.1007/s11098018-1151-2.

Stalnaker, R. (1984). Inquiry. Cambridge: Cambridge University Press.

Stalnaker, R. C. (2006). Logics of knowledge and belief. Philosophical Studies, 128, 169-199.

Sylvan, R. (1989). Relational semantics for all Lewis, Lemmon and Feys modal logics and most notably for systems between S0.3 and S1. The Journal of Nonclassical Logic, 6, 19-40.

Tang, W. H. (2018). In defence of single premise closure. Philosophical Studies, 175, 1887-1900.

Tuomela, R. (2004). Group knowledge analyzed. Episteme, 1, 109-127.

van der Hoek, W. (1996). Systems for knowledge and belief. Journal of Logic and Computation, 3, $173-195$.

von Kutschera, F. (1976). Einfuihrung in die intensionale Semantik. Berlin: W. de Gruyter.

Williamson, T. (1994). Vagueness. London: Routledge.

Williamson, T. (2000). Knowledge and its limits. Oxford: Oxford University Press.

Williamson, T. (2015). A note on gettier cases in epistemic logic. Philosophical Studies, 172, 129-140.

Publisher's Note Springer Nature remains neutral with regard to jurisdictional claims in published maps and institutional affiliations. 